\title{
26. TRIASSIC MARINE OSTRACODES OF THE AUSTRALIAN MARGIN (HOLES 759B, 760B, 761C, 764A, AND 764B) ${ }^{1}$
}

\author{
Françoise Dépêche² and Sylvie Crasquin-Soleau ${ }^{3}$
}

\begin{abstract}
Sixty-five species of benthic ostracodes have been discovered in the Triassic sediments of Ocean Drilling Program Leg 122, drilled on the northwestern margin of Australia. Known species were found in the samples studied from the upper Norian-Rhaetian at Holes 759B and 760B and from the Rhaetian at Holes 761C, 764A, and 764B. A large part of material of the recovered ostracodes belong to taxa that are related to ostracodes described in the Tethyan province. Seven species are known from northwestern Europe and five from Iran. Ogmoconcha and Rhombocythere, which are stratigraphically important genera in northern Europe, extend into the Tethyan province. Species of Ogmoconcha are present in Holes 760B, 764A, and 764B. The highly ornate baidiids of the Alpine Tethyan province are dominant in Hole 761C. The affinities with the fauna of Iran noted by Kristan-Tollmann are corroborated by the presence of the genera Mostlerella, Hiatobairdia, and other bairdiids.
\end{abstract}

\section{INTRODUCTION}

Leg 122 was drilled on the northwestern margin of Australia on the Exmouth Plateau, which is a rifted and subsided offshore fragment of a continental margin. More than $8 \mathrm{~km}$ of Phanerozoic sediments have been discovered that contain the history of vertical movements and sea-level fluctuations on this southern Tethyan margin. We recovered $3.5 \mathrm{~km}$ of Carnian to Holocene sediments at six sites. Drilling on Leg 122 had five main objectives:

1. To study the different phases of rifting and the sedimentary and paleoenvironmental developments from a young to mature ocean;

2. To test sequence stratigraphy models and eustatic curves;

3. To improve the chronostratigraphy of the Carnian to Cretaceous ages;

4. To analyze anoxic sediments from the Jurassic and Cretaceous; and

5. To retrieve sediments from the Cretaceous/Tertiary boundary.

Five holes extend into the Upper Triassic (Holes 759B, $760 \mathrm{~B}, 761 \mathrm{C}, 764 \mathrm{~A}$, and 764B). These are the oldest sediments drilled since the beginning of the Deep Sea Drilling Project (DSDP) and Ocean Drilling Program (ODP). These sites are located on the Wombat Plateau on the northern part of the Exmouth Plateau, adjacent to the oceanic crust of the Argo Abyssal Plain (Fig. 1). One km of Triassic sediments were recovered. These sediments contain foraminifers, calcareous nannofossils, ostracodes, and palynoflora. Here, we pay particular attention to ostracodes found in the four sites. The first discovery of Triassic ostracodes in ODP cores is an important contribution to the knowledge of Triassic ostracodes in the eastern part of the Tethys sea.

\footnotetext{
${ }^{1}$ von Rad, U., Haq, B. U., et al., 1992. Proc. ODP, Sci. Results, 122: College Station, TX (Ocean Drilling Program).

2 Laboratoire de Micropaléontologie, Département de Géologie Sédimentaire, Université P. et M. Curie, U.R.A. CNRS 1315, Paris, France.

${ }^{3}$ Département de Géologie Sédimentaire, Université P. et M. Curie, U.R.A. CNRS 1315, Paris, France.
}

\section{METHODS AND PROCEDURES}

Seventy-four samples have been studied ( 28 in Hole $759 \mathrm{~B}$, 17 in Hole $760 \mathrm{~A}, 16$ in Hole 760B, 7 in Hole 761C, 2 in Hole 764A, and 4 in Hole 764B). Sixty-one samples of unconsolidated sediments were washed under water and massive rocks were broken up by hot acetylase (Lethiers and Crasquin, 1988). More than 1,000 specimens were isolated from 14 fossiliferous samples.

Sixty-five species belonging to 27 genera have been studied under optical stereomicroscope and photographed by scanning electron microscopy.

Fossils are relatively scarce but well preserved, usually as carapaces. A few dissociated valves from collections in Sections $122-760 \mathrm{~B}-8 \mathrm{R}-3$ and $122-761 \mathrm{C}-31 \mathrm{R}-3$ have preserved hinge structures and adductor muscle-attachment scars.

\section{TRIASSIC OSTRACODES OF LEG 122}

The ostracodes from Leg 122 resemble the Alpine Triassic fauna in Europe, Israel, Jordan, Iran, Pakistan, the Himalayas, China, Timor, Papua New Guinea, Australia, New Zealand, and North America, as shown in the references (see, for example, Urlichs, 1972; Bolz, 1969, 1970, 1971a, 1971b; von Kollman, 1960, 1963; Kozur, 1968, 1970a, 1970b, 1971a, 1971b, 1971c, 1972; Bunza and Kozur, 1971; and Kristan-Tollmann, 1970, 1971, 1972, 1973, 1978, 1979, 1980, 1982, 1986, 1988). We also compared Leg 122 specimens to the fauna of Great Britain (Anderson, 1964; Bate, 1978), Spain (Kozur et al., 1974), Israel (Sohn, 1968), Jordan (Basha, 1982), Iran (Kristan-Tollmann, 1980), Pakistan (Sohn, 1970), China (Kristan-Tollmann, 1982), the Himalayas (Kristan-Tollmann et al., 1987), Timor (KristanTollmann et al., 1987), Alaska (Sohn, 1987; Kristan-Tollmann and Tollmann, 1983), Papua New Guinea, Australia, and New Zealand (Kristan-Tollmann, 1986).

Age determination is based on the following illustrated species:

Species described by Anderson (1964) as Hungarella are referred herein to Ogmoconcha.

Rhombocythere penarthensis Anderson (1964) (Pl. 4, Fig. 12): Norian-Rhaetian of Wales, Great Britain, and Germany (Bate, 1978).

Cytherella acuta Urlichs (1972) (PI. 3, Figs. 4, 5, and 8): upper Norian-Rhaetian of Germany (Urlichs, 1972); Rhaetian of Iran and Australia (Kristan-Tollmann et al., 1979, 1986). 


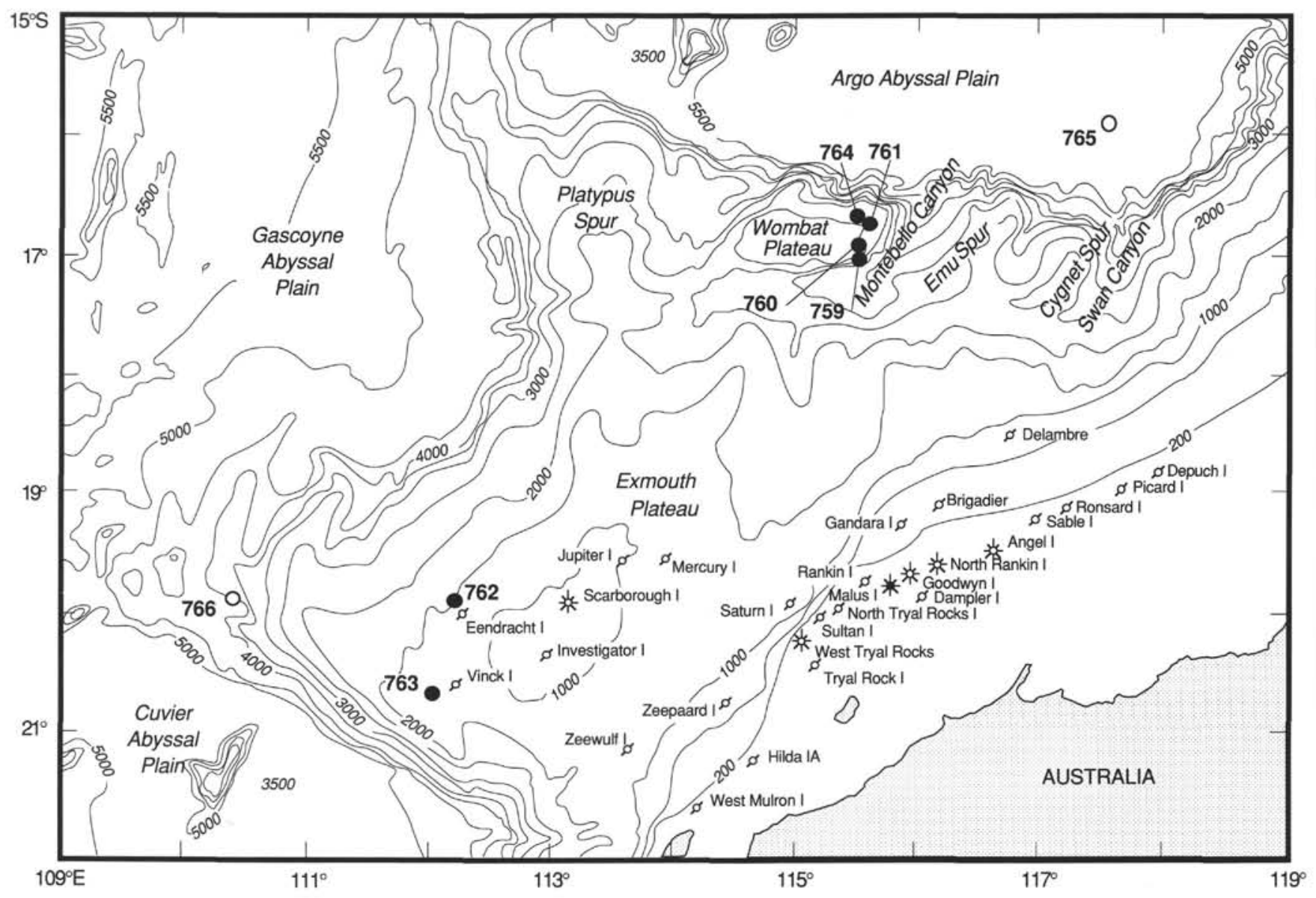

Figure 1. Location map of Leg 122 Sites 759, 760, 761, and 764.

Ogmoconcha martini (Anderson, 1964) (Pl. 4, Figs. 8-10, 15): upper Norian of England and Germany (Anderson, 1964; Will, 1969; Urlichs, 1972) and Rhaetian of Iran (KristanTollmann, 1980).

Nodobairdia mammilata Kollmann (1963) (PI. 2, Figs. 1-4): Ladinian-Rhaetian of the Alps, Austria (Kollmann, 1963; Bolz, 1971; Kristan-Tollmann, 1971, 1973, 1978) and Rhaetian of Iran and Australia (Kristan-Tollmann, 1980, 1986).

Cytherelloidea unicostata Bolz (1970) (PI. 3, Figs. 1-2): Norian-Rhaetian of Alps, Austria (Bolz, 1970).

Hiatobairdia subsymmetrica subsymmetrica Kristan-Tollmann (1970) (Pl. 1, Fig. 1): Rhaetian of Alps, Austria, and Iran (Kristan-Tollmann, 1970, 1980).

Ogmoconcha cf. reticulata Kristan-Tollmann (1980) (PI. 4, Figs. 4-6): Rhaetian of Iran (Kristan-Tollmann, 1980).

Ogmoconcha owthorpensis (Anderson, 1964): Rhaetian of Great Britain (Anderson, 1964).

Ogmoconcha bristolensis (Anderson, 1964) (Pl. 4, Fig. 3): Rhaetian of Great Britain (Anderson, 1964).

Judahella galli Kozur and Bolz (1971): upper Rhaetian of Mühlgraben (Austria).

Sixteen species compare to material described by previous authors. Most of the present material seems to be new. At least 17 species will be described in a later paper. At this time, several questions about the systematic descriptions of this new material remain unanswered.

\section{HOLE SUMMARIES}

All information on sites are from Haq, von Rad, O'Connell, et al. (1990).
Table 1. Ostracode distribution in Hole 759B.

\begin{tabular}{|c|c|c|c|c|}
\hline \multirow[t]{2}{*}{ Species } & \multicolumn{4}{|c|}{ Sections $122-759 \mathrm{~B}-$} \\
\hline & $-23 R-1$ & $-16 R-1$ & $-13 R-1$ & $-11 \mathrm{R}-2$ \\
\hline Acratia sp. & $\mathrm{X}$ & & & \\
\hline Gruendelicythere? sp. & & $\mathrm{X}$ & & $\mathrm{X}$ \\
\hline Acratidiae indet. & & $\mathrm{X}$ & & $\mathrm{X}$ \\
\hline Nodobairdia mammilata & & & $\mathrm{X}$ & \\
\hline Trebelina (Ptychobairdia) sp.1 & & & $\mathrm{X}$ & \\
\hline Judahella n.sp. I? & & & $x$ & \\
\hline Kerocythere? n.sp.2 & & & $\mathrm{X}$ & \\
\hline Simeonella? sp.1 & & & $\mathrm{X}$ & \\
\hline Cytherella acuta & & & $\mathrm{X}$ & $\mathrm{X}$ \\
\hline Kerocythere? sp.3 & & & & $\mathrm{X}$ \\
\hline Age & Carnian? & Norian & to & Rhaetian \\
\hline
\end{tabular}

\section{Hole 759B (Table 1)}

This is the southernmost site studied $\left(16^{\circ} 57.27^{\prime} \mathrm{S}\right.$, $115^{\circ} 33.61^{\prime} \mathrm{E}$, water depth $2091.9 \mathrm{~m}, 0-310$ meters below sea floor, or mbsf). Twenty-eight samples were studied; four of them yielded ostracodes. Ten species belonging to ten genera were recorded. Cytherella acuta Urlichs (1972) indicates that Sections 122-759B-11R-2 and 122-759B-3R-1 are late Norian to Rhaetian in age. We lack specimens to confirm a Carnian age for Section 122-759B-23R-1. With the exception of $N$. mammilata Kollmann, all other listed species may be new.

\section{Hole 760B (Table 2)}

This hole was drilled just north of Hole $759 \mathrm{~B}\left(16^{\circ} 55.32^{\prime} \mathrm{S}\right.$, $115^{\circ} 32.48^{\prime}$, water depth $1969.7 \mathrm{~m}$ ). It is subdivided into two parts: Hole $760 \mathrm{~A}$ from 0 to 284.9 mbsf (17 samples studied) yielded no ostracodes and Hole $760 \mathrm{~B}$ from 280 to $510 \mathrm{mbsf}$ (16 
Table 2. Ostracode distribution in Hole 760B.

\begin{tabular}{|c|c|c|}
\hline \multirow[t]{2}{*}{ Species } & \multicolumn{2}{|c|}{ Sections 122-760B } \\
\hline & $-9 \mathrm{R}-2$ & $-8 \mathrm{R}-3$ \\
\hline Kerocythere? n.sp.2 & $\mathrm{X}$ & \\
\hline Ogmoconcha cf.blakei & $\mathrm{X}$ & \\
\hline Reubenella n.sp.1 & $\mathrm{X}$ & $\mathrm{X}$ \\
\hline Judahella n.sp.i & $\mathrm{X}$ & $\mathrm{X}$ \\
\hline Rhombocythere penarthensis & & $\mathrm{X}$ \\
\hline Judahella sp. & & $\mathrm{X}$ \\
\hline Kerocythere? n.sp.I & & $\mathrm{X}$ \\
\hline Biardiacypris cf. triassica & & $\mathrm{X}$ \\
\hline Triebelina (Ptychobairdia) sp.I & & $\mathrm{X}$ \\
\hline Triebelina (Ptychobairdia) sp.2 & & $\mathrm{X}$ \\
\hline Nodobairdia mammilata & & $\mathrm{X}$ \\
\hline Reubenella sp. & & $\mathrm{X}$ \\
\hline Liasina cf.lanceolata & & $\mathrm{X}$ \\
\hline Ogmoconcha martini & & $\mathrm{X}$ \\
\hline Reubenella n.sp.2 & & $\mathrm{X}$ \\
\hline Reubenella sp.A & & $\mathrm{X}$ \\
\hline Ogmoconcha cf.reticulata & & $\mathrm{X}$ \\
\hline Ogmoconcha owthorpensis & & $\mathrm{X}$ \\
\hline Age & $\begin{array}{r}\text { Upper } \\
\text { Rh }\end{array}$ & $\begin{array}{l}\text { Norian } \\
\text { tian }\end{array}$ \\
\hline
\end{tabular}

Tabl.2

samples studied) contained ostracodes in two samples. Twohundred thirty-eight specimens were encountered in Sections 122-760B-9R-2 and 122-760B-8R-3. Eighteen taxa belonging to nine genera were recorded. Ogmoconcha martini (Anderson, 1964) and Rhombocythere penarthensis Anderson (1964) suggest a late Norian to Rhaetian age. Consequently, Sections $122-760 \mathrm{~B}-8 \mathrm{R}-3$ and $122-760 \mathrm{~B}-9 \mathrm{R}-3$ may be slightly younger than the Norian age attributed by Haq, von Rad, O'Connell, et al. (1990).

\section{Hole 761C (Table 3)}

This site is located north of Site $760\left(16^{\circ} 44.28^{\prime} \mathrm{S}\right.$, $115^{\circ} 32.10^{\prime} \mathrm{E}$, water depth $2167.9 \mathrm{~m}$ ). The part of the hole we examined is from 260 to $436.7 \mathrm{mbsf}$. Seven samples have been studied; five contain ostracodes. Numerous specimens were recovered from Sections 122-761C-31R-3, 122-761C-28R-CC, 122-761C-25R-CC, 122-761C-24R-3, and 122-761C-23R-1. We found 33 species belonging to 19 described genera, and one to a new genus. Section 122-761C-31R-3 contains Ogmoconcha owthorpensis (Anderson, 1964), which is found in sediments of the middle Rhaetian of Europe and Cytherella acuta Urlichs (1972) (upper Norian to middle Rhaetian). Section 122-761C-25R-CC contains Ogmoconcha bristolensis (Anderson, 1964) from the middle Rhaetian. Nodobairdia mammilata Kollmann (1963) is present in Section 122-761C-24R-3. Hiatobairdia subsymmetrica subsymmetrica Kristan-Tollmann (1980) is observed in Section 122-761C-35R-1 (Rhaetian). A Rhaetian age earlier attributed to those sediments seems to be confirmed for Sections 122-761C-31R-3 and 122-761C-25RCC.

\section{Holes 764A and 764B (Table 4)}

This is the northernmost site of Leg $122\left(19^{\circ} 53.23^{\prime} \mathrm{S}\right.$, $112^{\circ} 15.24^{\prime} \mathrm{E}$, water depth $2698.6 \mathrm{~m}$ ). This site is subdivided into two parts: Hole $764 \mathrm{~A}$ from 0 to 70 mbsf (two samples, ostracodes in one of them) and Hole 764B from 40 to 294.5 mbsf (four samples, ostracodes in all of them). Ostracodes assemblages are dominated by the species Ogmoconcha martini (Anderson, 1964), Cytherella acuta Urlichs (1972), and Cytherelloidea? unicostata Bolz (1970a). Although Judahella galli Kozur and Bolz (1971) was described in the Carnian of Austria; this fauna indicates a Rhaetian age. Ogmoconcha bristolensis (Anderson, 1964) and Ogmoconcha owthorpensis (Anderson, 1964) in Sections 122-764B-31R-5 and 122-764B6R-1 are characteristic species of the middle Rhaetian. This faunal assemblage still suggests a Rhaetian age, and more accurately a middle Rhaetian age. Except for the new genus sp. 1 (Table 4), the following taxa are closely related to species described from the Upper Triassic (Carnian to Rhaetian):

Table 3. Ostracode distribution in Hole 761C.

\begin{tabular}{|c|c|c|c|c|c|}
\hline \multirow[t]{2}{*}{ Species } & \multicolumn{5}{|c|}{$-122-761 \mathrm{C}$} \\
\hline & 3IR-3 & $28 \mathrm{R}-\mathrm{CC}$ & $25 \mathrm{R}-\mathrm{CC}$ & $24 \mathrm{R}-3$ & 23R-1 \\
\hline Cytherella acuta & $\mathrm{X}$ & & & & \\
\hline Lophocythere? sp.1 & $\mathrm{X}$ & & & & \\
\hline Ogmoconcha owthorpensis & $\mathrm{X}$ & & & & \\
\hline Judahella n.sp.2 & $\mathrm{X}$ & & & $\mathrm{X}$ & \\
\hline Kerocythere? n.sp.1 & $\mathrm{X}$ & & & $\mathrm{X}$ & \\
\hline Bairdiacypris cf.triassica & $\mathrm{X}$ & & $\mathrm{X}$ & $\mathrm{X}$ & $\mathrm{X}$ \\
\hline Bairdia sp.C & & $\mathrm{X}$ & & & \\
\hline Ogmoconcha bristolensis & & & $\mathrm{X}$ & & \\
\hline Cytherelloidea? unicostata & & & $\mathrm{X}$ & $\mathrm{X}$ & \\
\hline Cornutobairdia aff.trinodosa & & & & $\mathrm{X}$ & \\
\hline Triebelina (Nodobairdia) n.sp. & & & & $\mathrm{X}$ & \\
\hline Triebelina (T.) aff.T.(T.) longiforme & & & & $\mathrm{X}$ & \\
\hline Triebelina (T.) aff.T.(T.)sp.498 & & & & $\mathrm{X}$ & \\
\hline Paracypris cf.redcarensis & & & & $\mathrm{X}$ & \\
\hline Bairdia sp. B & & & & $\mathrm{X}$ & \\
\hline Liasina cf.lanceolata & & & & $\mathrm{X}$ & \\
\hline Nodobairdia mammilata & & & & $\mathrm{X}$ & \\
\hline Tutkevichinella aff.keupera & & & & $\mathrm{X}$ & \\
\hline Polycope sp. & & & & $\mathrm{X}$ & \\
\hline Bythocypris sp.B & & & & $\mathrm{X}$ & $\mathrm{X}$ \\
\hline Lobobairdia cf.salinaria & & & & X & $\mathrm{X}$ \\
\hline Bairdia n.sp. cf.B. cassiana & & & & $\mathrm{X}$ & $\mathrm{X}$ \\
\hline Judahella sp. $A$ & & & & & $X$ \\
\hline Praeschuleridea? n.sp.1 & & & & & $\mathrm{X}$ \\
\hline Bairdia n.sp.2 & & & & & $\mathrm{X}$ \\
\hline Bairdia cf.anisica & & & & & $\mathrm{X}$ \\
\hline Bythocypris sp.A & & & & & $\mathrm{X}$ \\
\hline Bairdiacypris sp.192 & & & & & $\mathrm{X}$ \\
\hline Paracypris sp.200 & & & & & $\mathrm{X}$ \\
\hline Kerocythere cf.hartmanni & & & & & $\mathrm{X}$ \\
\hline Bairdia sp. $A$ & & & & & $\mathrm{X}$ \\
\hline n gen. n. sp. 2 & & & & & $\mathrm{X}$ \\
\hline Hiatobairdia subsymmetrica & & & & & $\mathrm{X}$ \\
\hline & & $\mathbf{R}$ & a & a $n$ & \\
\hline
\end{tabular}


Table 4. Ostracode distribution in Holes 764A and 764B.

\begin{tabular}{|c|c|c|c|c|c|}
\hline \multirow[t]{2}{*}{ Species } & \multicolumn{3}{|c|}{ Sections -122- } & \multicolumn{2}{|l|}{ 764A-B } \\
\hline & $-31 R-5$ & $-8 R-1$ & T-7R-1 & T-8R-CC & $1-6 R-1$ \\
\hline Kinkelinalla? sp.1 & $\mathrm{x}$ & & & & \\
\hline Judahella ? sp.1 & $x$ & & & & \\
\hline Cytherelloidea?unicostata & $\mathrm{X}$ & & & & \\
\hline Noricythere n.sp. & $\mathrm{X}$ & & & & \\
\hline Pontocypris cf.richardsoni & $\mathrm{X}$ & & & & \\
\hline Cytherella acuta & $\mathrm{X}$ & & & & \\
\hline Ogmoconcha martini & $X$ & & & & \\
\hline Bairdia n.sp.219 & $\mathrm{X}$ & & & & \\
\hline Paracypris cf.redcarensis & $\mathrm{X}$ & & & & \\
\hline Judahella galli & $X$ & & & & \\
\hline Judahella n.sp.2 & $x$ & & & $x$ & \\
\hline Ogmoconcha bristolensis & $\mathrm{X}$ & & & & $\mathrm{X}$ \\
\hline Kerocythere? n.sp.1 & $\mathrm{X}$ & & & & $\mathrm{X}$ \\
\hline Ogmoconcha owthorpensis & $\mathrm{X}$ & $\mathrm{X}$ & & $X$ & $\mathrm{X}$ \\
\hline Ogmoconcha cf.reticulata & & $\mathrm{X}$ & & & \\
\hline Luckevichinella sp.1 & & $\mathrm{x}$ & & & \\
\hline n. gen. n.sp. 2 & & $\mathrm{x}$ & & $\mathrm{X}$ & \\
\hline Bairdia sp.2 & & $\mathrm{X}$ & & & \\
\hline Bairdia n.sp.1 & & & $\mathrm{X}$ & & \\
\hline Paracypris sp.1 & & & $\mathrm{X}$ & & \\
\hline Bairdiacypis cf.triassica & & & & $X$ & \\
\hline Kerocythere? sp.3 & & & & $\mathrm{X}$ & \\
\hline n. gen. n. sp. 1 & & & & $\mathrm{X}$ & \\
\hline Praeschuleridea? sp.2 & & & & $\mathrm{X}$ & \\
\hline Bairdia sp. A & & & & & $\mathrm{X}$ \\
\hline Age & & & $\mathrm{Rh} \mathrm{a}$ & tian & \\
\hline
\end{tabular}

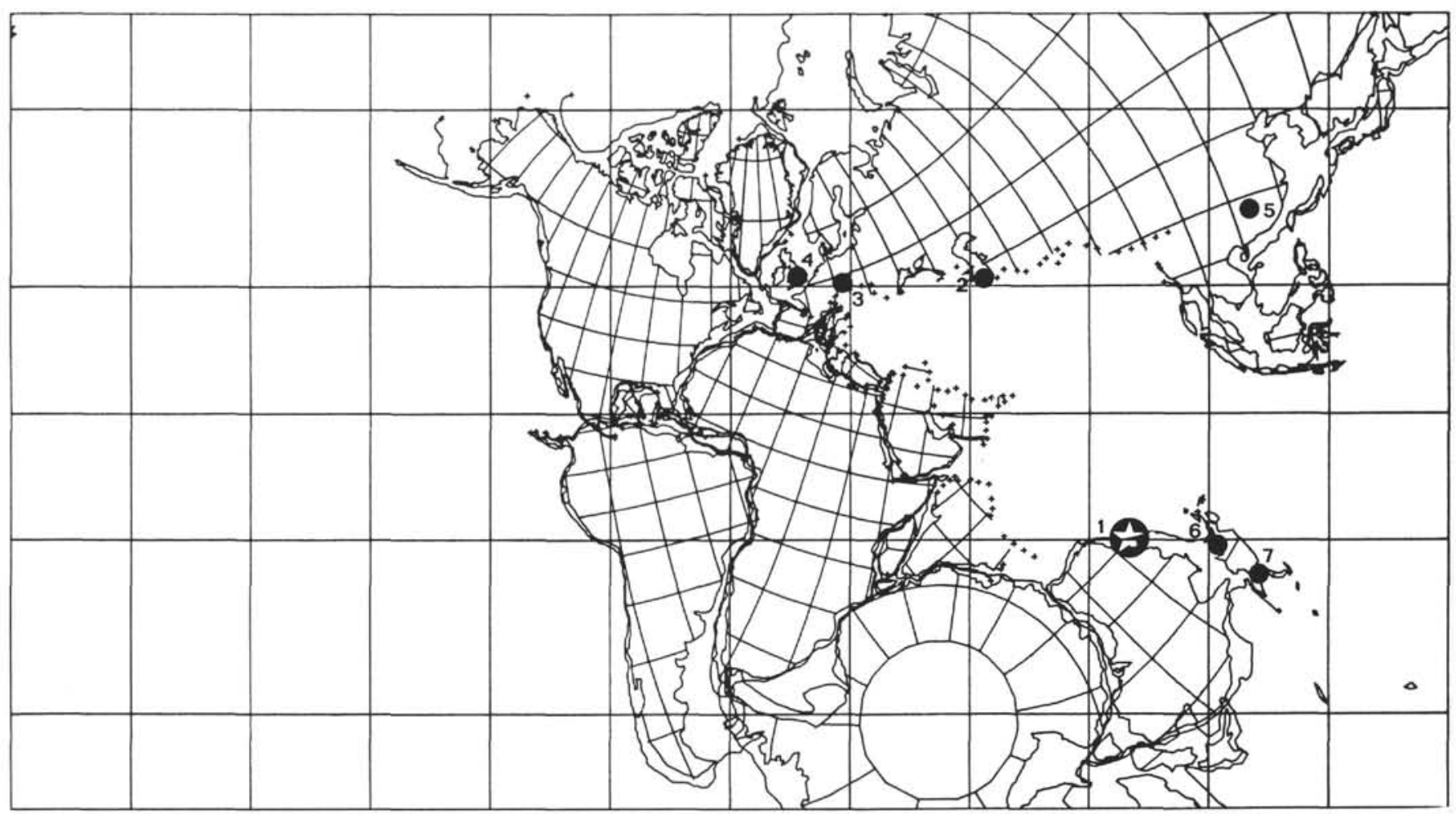

Figure 2. Paleogeographic map of the latest Triassic (Rhaetian) (Mercator, $N=67$, alpha- $\left.95=6^{\circ} 1\right)$, with location of ostracode sites $(1=\mathrm{Leg}$ 122, 2 = Iran, 3 = Alps, $4=$ Great Britain, $5=$ China, $6=$ Papua New Guinea, and $7=$ New Zealand).

Paracypris cf. richardsoni Anderson (1964), Paracypris cf. redcarensis (Blake, 1876), Ogmoconcha owthorpensis (Anderson, 1964), Bairdiacypris cf. triassica Kozur (1971), and Judahella galli Kozur and Bolz (1971).

\section{PALEOECOLOGY}

Analysis of faunal composition allows us to identify paleoenvironment. The occurrence of the euryhaline ostracodes
Rhombocythere penarthensis and Simeonella? may indicate brackish hypersaline conditions. Species of typically marine genera, such as all forms shown in the figures, are found together with Rhombocythere in Sections 122-760B-9R-2 and $122-760 \mathrm{~B}-8 \mathrm{R}-3$ and suggest mixing with open ocean. These forms are present with sculptured bairdiids characteristic of shallow, warm water connected to coral reefs. Smooth bairdiids expand in the warm, calm water of the littoral zone, 
while the dominance of Ogmoconcha in Holes 760B, 764A, and $764 \mathrm{~B}$ indicates somewhat deeper water in open-marine conditions.

\section{CONCLUSIONS}

Sixty-five species of benthic ostracodes have been recognized in the studied samples of Leg 122 Triassic sediments. The majority of taxa are new and have to be described in another paper. Identified species indicate a late NorianRhaetian age for samples of Holes 759B and 760B and a Rhaetian age for Hole 761C, 764A, and 764B. Several species are common in the five holes. The ostracode assemblages are more diversified in Holes 761C, 764A, and 764B. Species of Ogmoconcha prevail in three holes (760B, 764A, and 764B); bairdiidae are dominant in Hole 761C. Species are consistent with those found in Australia (Kristan-Tollmann, 1986), Iran (Kristan-Tollmann, 1980), and Europe. The position of Leg 122 on a paleogeographical map (Fig. 2) shows that there are relations in ostracode communities of the southern and western margins of the Tethys during the Late Triassic. The presence of similar ostracodes assemblages along Triassic Tethyan margins are very likely due to climatic zonation and to current circulation (Kristan-Tollmann and Tollmann, 1983).

\section{ACKNOWLEDGMENTS}

We want to acknowledge ODP France and URA 1315 for financial support and Bruno Galbrun (CNRS, Paris) for his help.

\section{REFERENCES}

Anderson, F. W., 1964. Rhaetic Ostracoda. Bull. Geol. Surv. G.B., 21:133-174.

Basha, S.H.S., 1982. Microfauna from the Triassic rocks of Jordan. Rev. Micropaleontol., 25:3-11.

Bate, R. H., 1978. The Trias. In Bate, R. H., and Robinson, E. (Eds.), A Stratigraphic Index of British Ostracoda. Geol. J. Spec. Iss., $8: 175-187$.

Bolz, H., 1969. Der "bairdopillate" Verschluss und Skulptur-Unterschiede bei Bairdien (Ostracoda) der alpinen Obertrias. Senckenbergiana Lethaea, 50:411-431.

1970. Einige Cytherelloidea-Arten (Ostrac.) aus der alpinen Ober trias. Senckenbergiana Lethaea, 51:239-263.

1971a. Die Zlambach-Schichten (alpine Obertrias) unter besonderer Berucksichtigung der Ostrakoden, 1: Ostrakoden der Zlambach-Schichten, besonders Bairdiidae. Senckenbergiana Lethaea, 52:129-283.

1971b. Late Triassic Bairdiidae and Healdiidae. Bull. Cent. Rech. Pau. SNPA, 5 (suppl.):717-745.

Bunza, G., and Kozur, H., 1971. Beitrage zur Ostracoden fauna der tethyalen Trias. Geol. Palaontol. Mitt. Ibk., 1:1-76.

Haq, B. U., von Rad, U., O'Connell, S., et al., 1990. Proc. ODP, Init. Repts., 122: College Station, TX (Ocean Drilling Program).

Kozur, H., 1968. Neue Ostracoden aus dem Röt und Muschelkalk des Germanischen Binnenbecken. Monatsber. Dtsch. Akad. Wiss. Berlin, 10:498-519.

1970a. Neue Ostracoden aus der germanischen Mittel und Obertrias. Geologie, 19:434-455.

1970b. Neue Ostracoden-Arten aus dem obersten Anis des Bakony-Hochlandes (Ungarn). Ber. Nat. Mad. Ver. Innsbruck, 58:384-428.

1971a. Die Bairdiacea des Trias. Teil. 1: Skulpturierte Bairdiidae aus Mitteltriassischen Flachwasse ablagerungen. Geol. Palaont. Mitt. Ibk., 1:1-27.

1971b. Die Bairdiacea der Trias.Teil 2: Skulpturierte Bairdiidae aus mitteltriassischen Teifschefablagerungen. Geol. Palaont. Mitt. Ibk, 1:1-21.

1971c. Die Bairdiacea der Trias. Teil 3: Einige neue Arten triassischer Bairdiacea und Bemerknngen zur Herkunft der Macrocyprididae (Cypridacea). Geol. Palaont. Mitt. Ibk., 1:119.
1972. Die Bedeutung triassischer Ostracoden für stratigraphische und paläoökologische Untersuchungen. Mitt. Ges. Geol. Bergbaustud, 21:632-660.

Kozur, H., Kampschuur, W., Mulder-Blanken, C.W.H., and Simon, O. J., 1974. Contribution to Triassic ostracode faunas of Betic zone (Southern Spain). Scripta Geol., 23:1-63.

Kristan-Tollmann, E., 1970. Einige neue Bairdien (Ostracoda) aus der alpinen Trias. Neues Jahrb. Geol. Palaeontol. Abh., 135:268-310. 1971. Weitere Beobachtungen an skulptierten Bairdiidae (Ostrac.) der alpinen Trias. Neues Jahrb. Geol. Palaeontol. Abh., 139:57-81.

1972. Die obertriadischen Arten der Ostracodengattung Kerocythere Kozur and Nicklas, 1970, und ihr stratigraphischer wert. Erdoel-Erdgas-Z., 88:43-49. 1973. Zur Ausbildung des Schliessmuskelfeldes bei triassichen Cytherellidae (Ostracoda). Neues Jahrb. Geol. Palaeontol. Monatsh., 6:351-373.

1978. Bairdiidae (Ostracoda) aus den Obertriassichen Cassianer Schichten der Ruones-Wiesen bei Covara in Süd Tyrol. Schriftenr. Erdwiss. Komm.-Österr. Akad. Wiss., 4: 77-104.

1979. Triassic ostracods in the Salzkammergut and Salzburg area. Limnol. Inst. Österr. Akad. Wiss., 6-28.

Kristan-Tollmann, E., 1980. Die Mikrofauna: Ostracoden. In KristanTollmann, E., Tollmann, A., and Hamedani, A., 1980. Beiträge zur kenntis der Trias von Persien; Zur Rhätfauna von Bagerabad bei Isfahan (Korallen, Ostracoden). Mitt. Österr. Geol. Ges., $76: 213-272$.

1982. Ostracoden aus dem Oberanis von Leidapo bei Guiyang in Südchina. Schriftenr. Erdwiss. Komm. Österr. Akad. Wiss., 5:121-176.

1982. Bemerkungen zur triadischen Ostracodengattung Mostlerella. Neues. Jahrb. Geol. Palaeontol. Monatsh., 5:560-572. 1986. Beobachtungen zur Trias am Sudostende der TethysPapua/New-Guinea, Australia, Neuseeland. Neues. Jahrb. Geol. Palaeontol. Monatsh., 4:201-222.

1988. Unexpected microfaunal communities within the Triassic Tethys. Geol. Soc. Spec. Publ. London, 37:213-223.

Kristan-Tollmann, E., and Tollmann, A., 1983. Tethysfaunenelemente in der Trias der USA. Mitt. Österr. Geol. Ges., 76:213-272.

Kristan-Tollmann, E., Tollmann, A., and Hamedani, A., 1980. Beiträge zur kenntis der Trias von Persien; Zur Rhätfauna von Bagerabad bei Isfahan (Korallen, Ostracoden). Mitt. Österr. Geol. Ges., 76:213-272.

Kristan-Tollmann, E., Barkham, S., and Gruber, B., 1987. Pötschenschichten, Zlambachmergel (Hallstätter Obertrias) und Liasfleckenmergel in Zentraltimor, nebst ihren Fanenelementen. Mitt. Österr. Geol. Ges., 80:229-285.

Lethiers, F., and Crasquin, S., 1988. Comment extraire des microfossiles à tests calcitiques de roches calcaires dures. Rev. Micropaleontol., 31:56-61.

Sohn, I. G., 1968. Triassic Ostracodes from Makhtesh Ramon. Bull. Geol. Surv. Isr., 44:1-71

1970. Early Triassic Marine Ostracodes from the Salt Range and Surghar Range, West Pakistan. In Kummel, B., and Teichert, T. (Eds.), Stratigraphy Boundary Problems: Permian and Triassic of Western Pakistan. Univ. Press Kansas, 193-206. 1987. Middle and Upper Triassic Marine Ostracoda from the Shublik Formation, Northeastern Alaska. U.S. Geol. Surv. Bull., 1664:C1-C47.

Urlichs, M., 1972. Ostracoden aus den Kössener Schichten und ihre Abhängigkeit von der Ökologie. Mitt. Ges. Geol. Bergbaustud. Wien, 21:661-710.

von Kollmann, K., 1960. Ostracoden aus der alpinen Trias Osterreichs. 1: Parabairdia n.g. und Ptychobairdia n.g. (Bairdiidae). Jahrb. Geol. Bundesanst Austria, 5:79-105.

1963. Ostracods from the Alpine triassic. II. Further Bairdiidae. Jahrb. Geol. Bundesanst Aust., 106:121-203.

Will, H. J., 1969. Untersuchungen zur Stratigraphie und Genese des Ober-Keupers in Northwest-Deutschland. Geol. Jahrb., Beih., $54: 1-240$.

Date of initial receipt: 12 June 1990

Date of acceptance: 25 June 1991

Ms 122B-177 

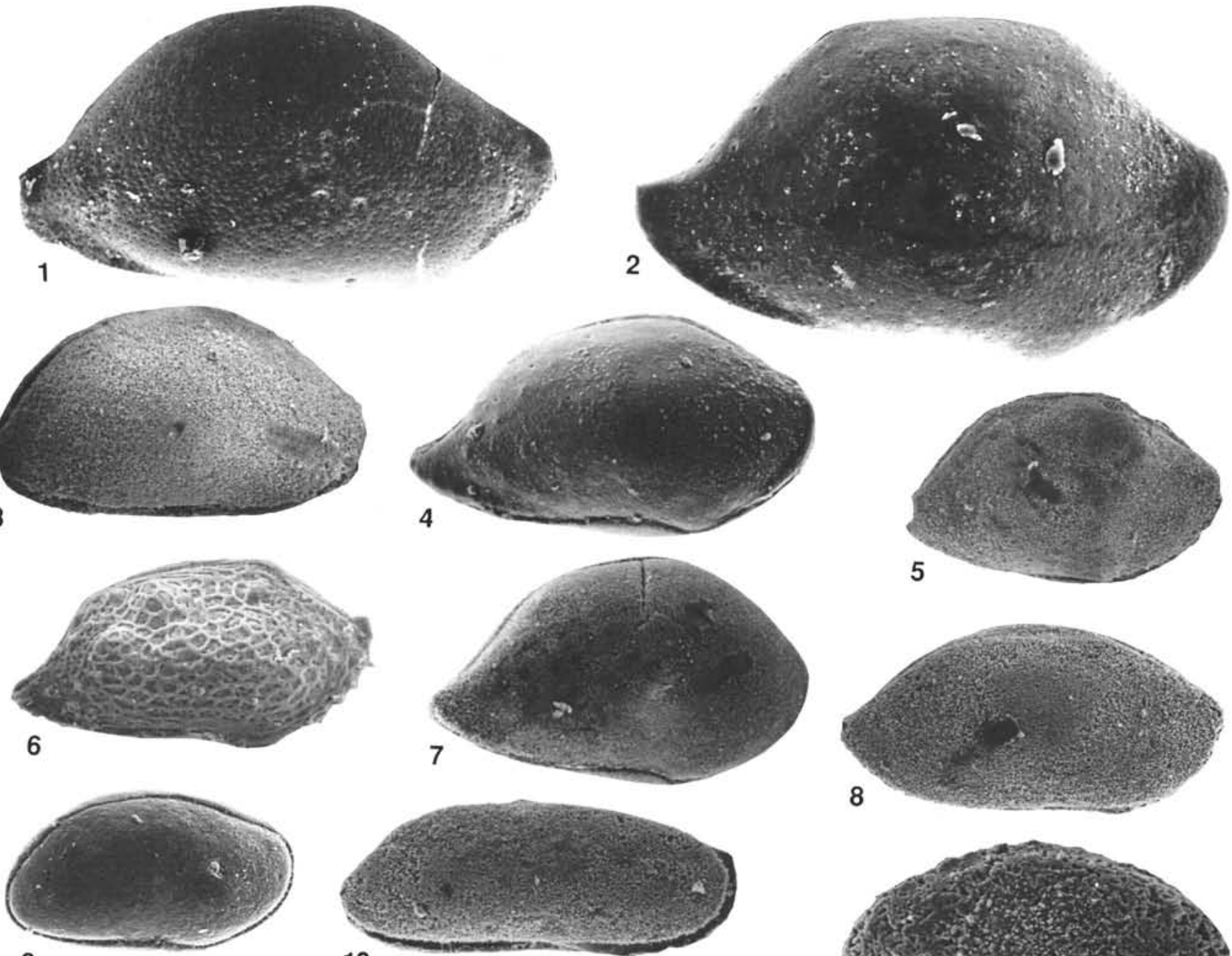

9

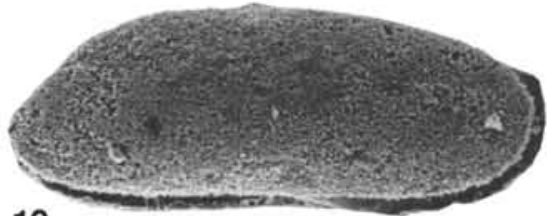

10
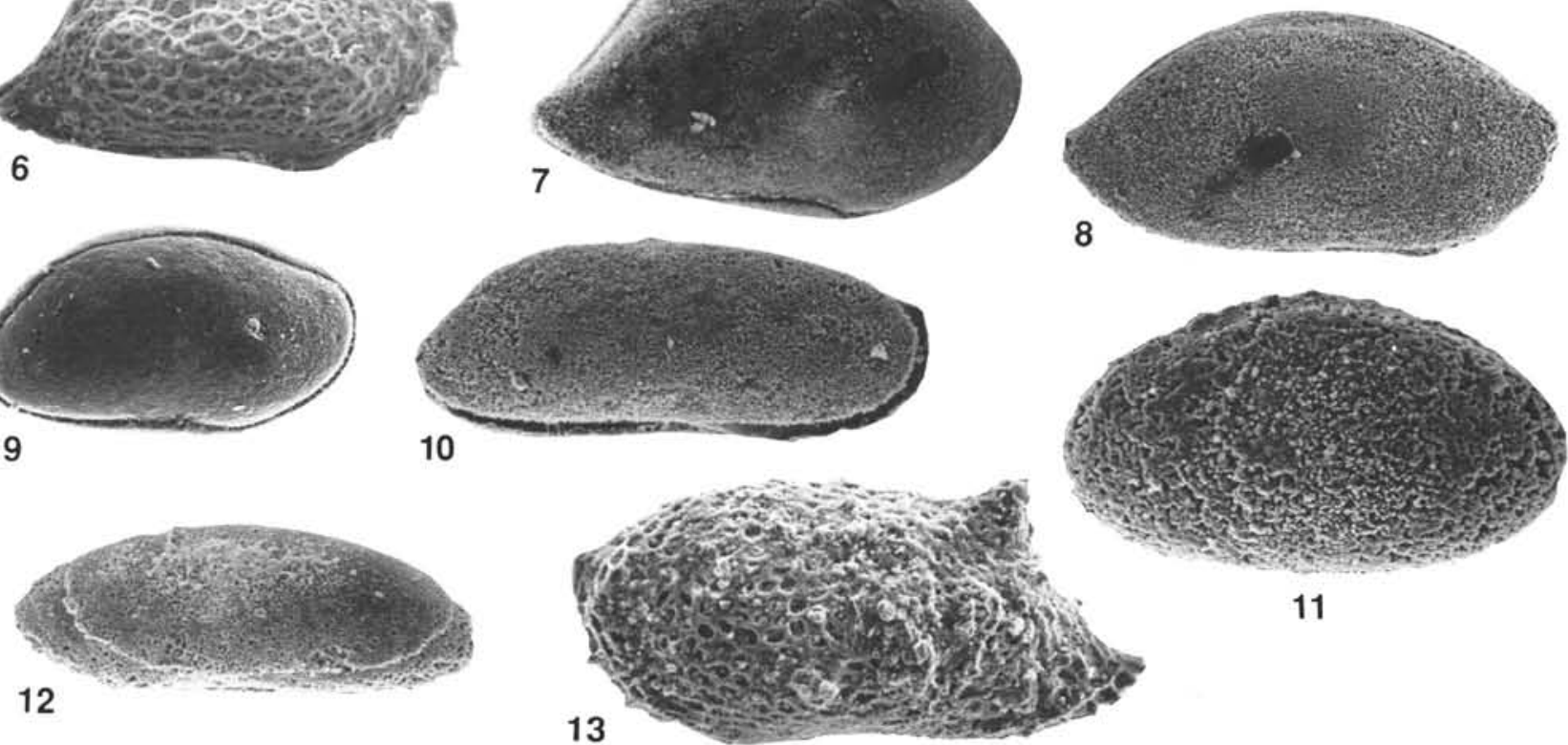

11

Plate 1. Magnification is $80 \times$ unless otherwise stated. 1. Hiatobairdia subsymmetrica subsymmetrica Kristan-Tollmann (1980), right view, Section 122-764C-23R-1. 2. Bairdia n. sp. cf. B. cassiana (Reuss, 1868), right view, 100×, Section 122-761C-24R-3. 3. Bairdia cf. B. anisica Kozur (1970), right view, 70×, Section 761C-23R-1. 4. Bairdia n. sp. cf. B. cassiana Reuss (1868), right valve, Section 122-761C-24R-1. 5. Bairdia n. sp. 2, right view, 50×, Section 122-761C-23R-1. 6. Bairdia n. sp. 219, right view, 110×, Section 122-764B-31R-5. 7. Bairdia sp. 2, right view, 100×, Section 122-764B-8R-1. 8. Bairdia sp. A, right view, 70×, Section 122-761C-23R-1. 9. Bairdiacypris cf. triassica Kozur (1971), right view, 50×, Section 122-761C-24R-3. 10. Bairdiacypris sp. 192, right view, 90×, Section 122-761C-23R-1. 11. Bythocypris sp. A, right view, $110 \times$, Section 122-761C-23R-1. 12. Bythocypris sp. B, right view, 70×, Section 122-761C-23R-1. 13. Cornutobairdia aff. trinodosa Kristan-Tollmann (1971), left view, 90×, Section 122-761C-24R-3. 

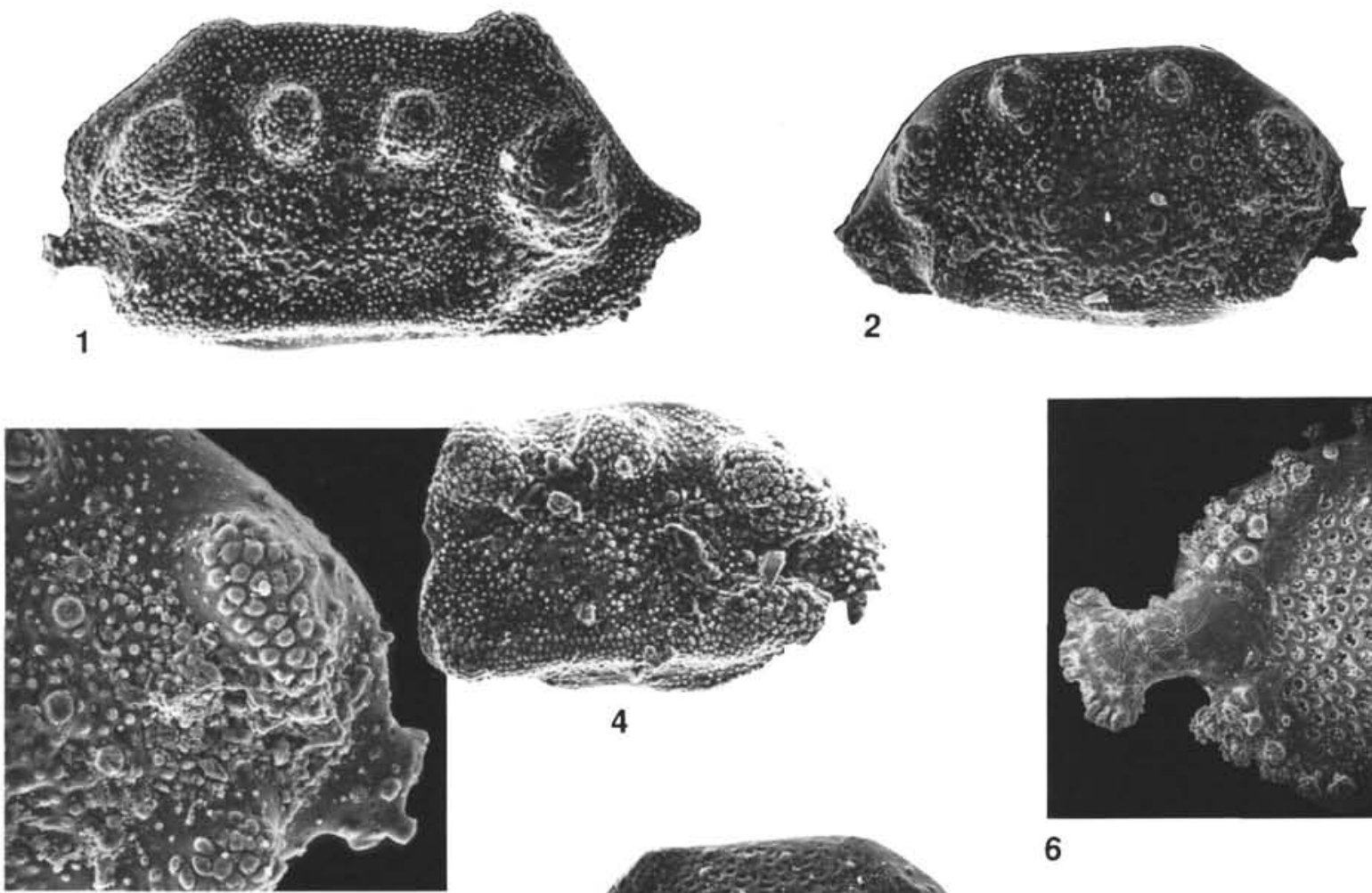

4

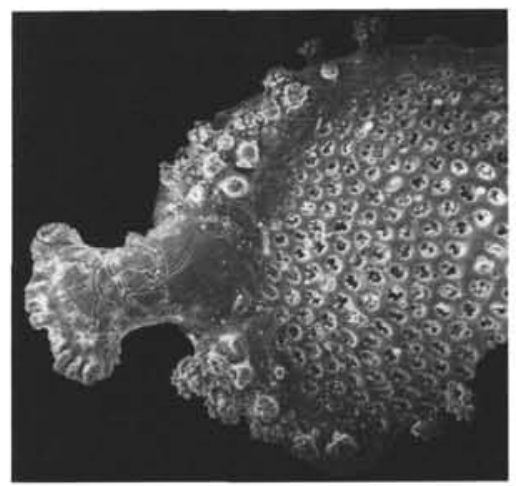

3

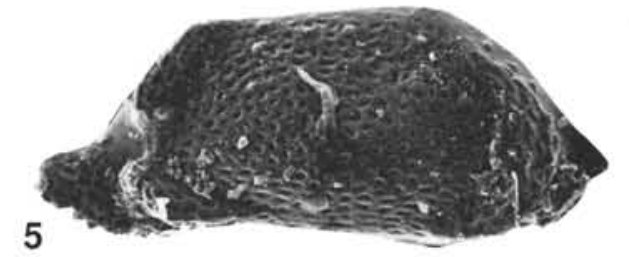

6
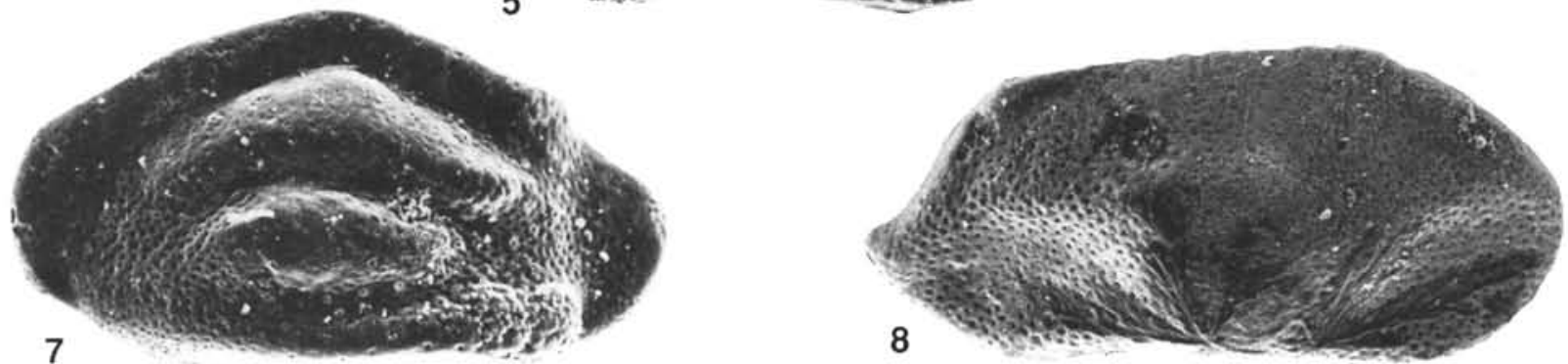

Plate 2. Magnification is $80 \times$ unless otherwise stated. 1-4. Nodobairdia mammilata Kollmann (1963); (1) left view, Section 122-760B-8R-3; (2) right view, Section 122-761C-24R-3; (3) right view (detail of Fig. 2, 150×); (4) right view, Section 122-760B-8R-3. 5. Triebelina (Triebelina) aff. T. longiforma Bolz (1971), right view, Section 122-760B-8R-3. 6. Triebelina sp. broken carapace, Section 122-760B-8R-3. 7. Triebelina (Ptychobairdia) sp. 1, right view, Section 122-760B-8R-3. 8. Triebelina (Triebelina) aff. Triebelina (Triebelina) sp. 498 sensu Kollmann (1963), right view, Section 122-761C-8R-3. 

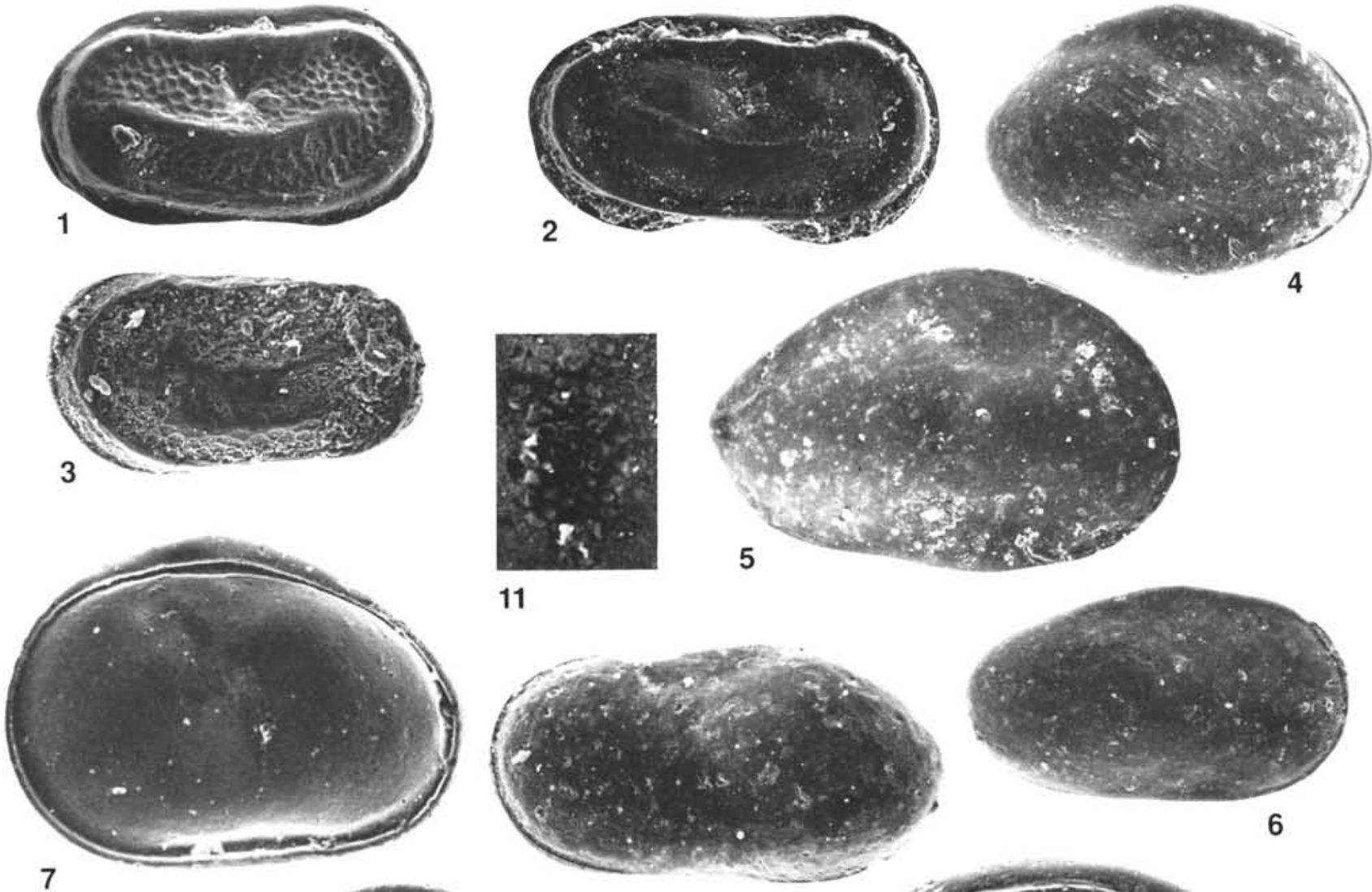

11
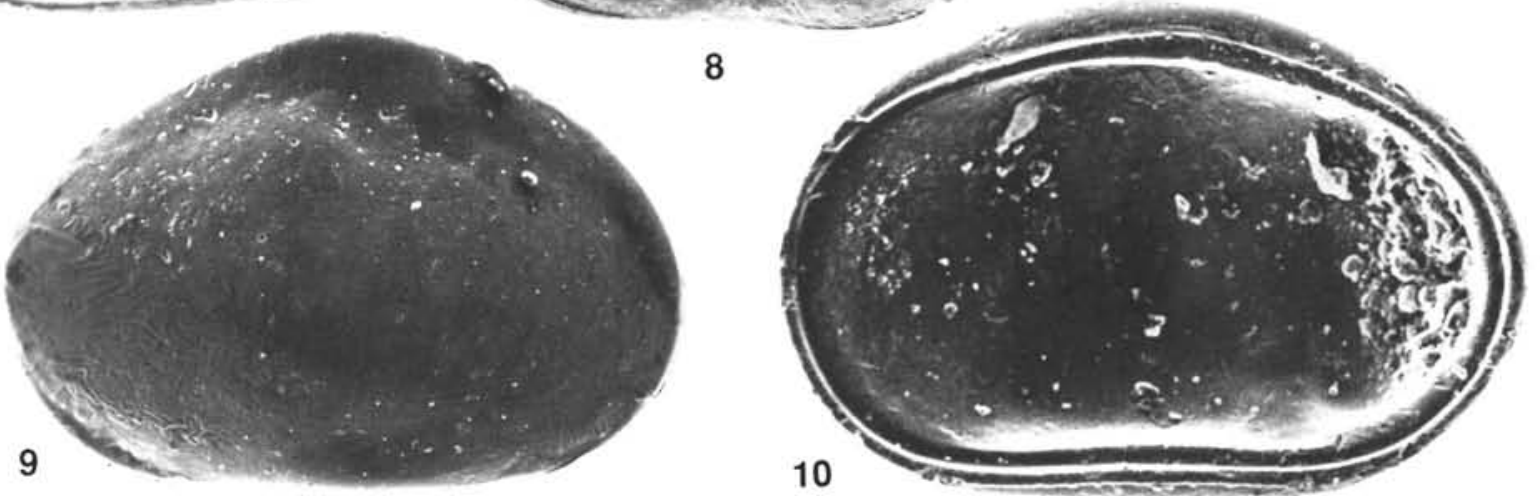

Plate 3. Magnification is $80 \times$ unless otherwise stated. 1, 2. Cytherelloidea? unicostata Bolz (1970); (1) right view, reticulate form, Section 122-764B-31R-5; (2) right view, smooth form, Section 122-761C-24R-3. 3. Cytherelloidea sp.?, left view, Section 122-761C-24R-3. 4, 5, (8?). Cytherella acuta Urlichs (1972), (4) right view, (5) left view, (8?) left view, Section 122-760B-8R-3. 6, 7, 9, 10. Reubenella n. sp. 1; (6) juvenile form, right view, (7) left view, (9) left view, (10) right internal view, Section 122-760B-8R-3. 11. Reubenella sp., adductor muscle scars, 300×, Section 122-760B-8R-3. 

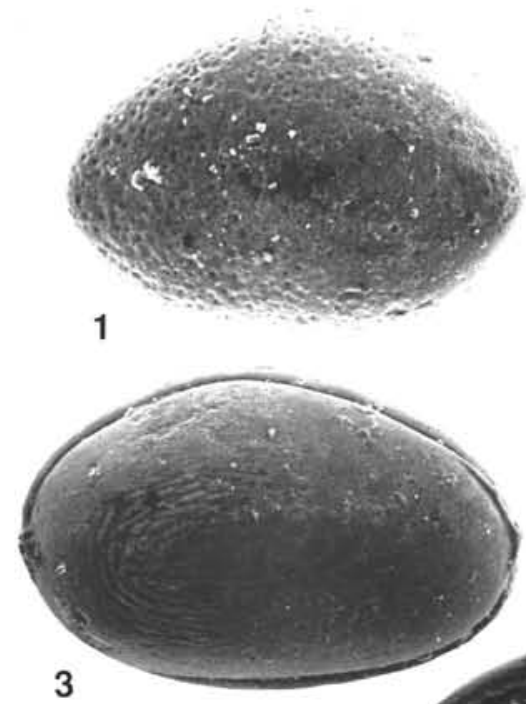

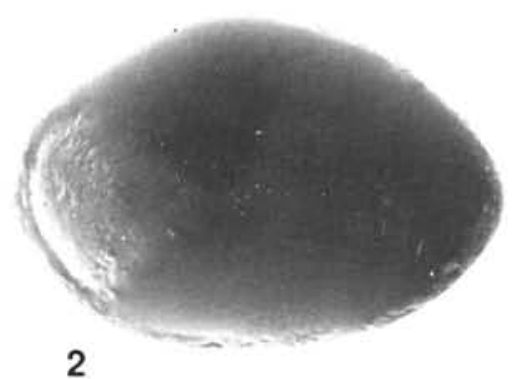

2

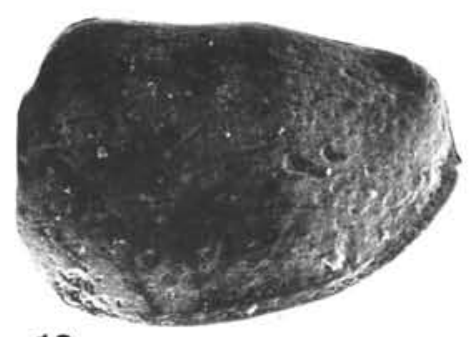

12

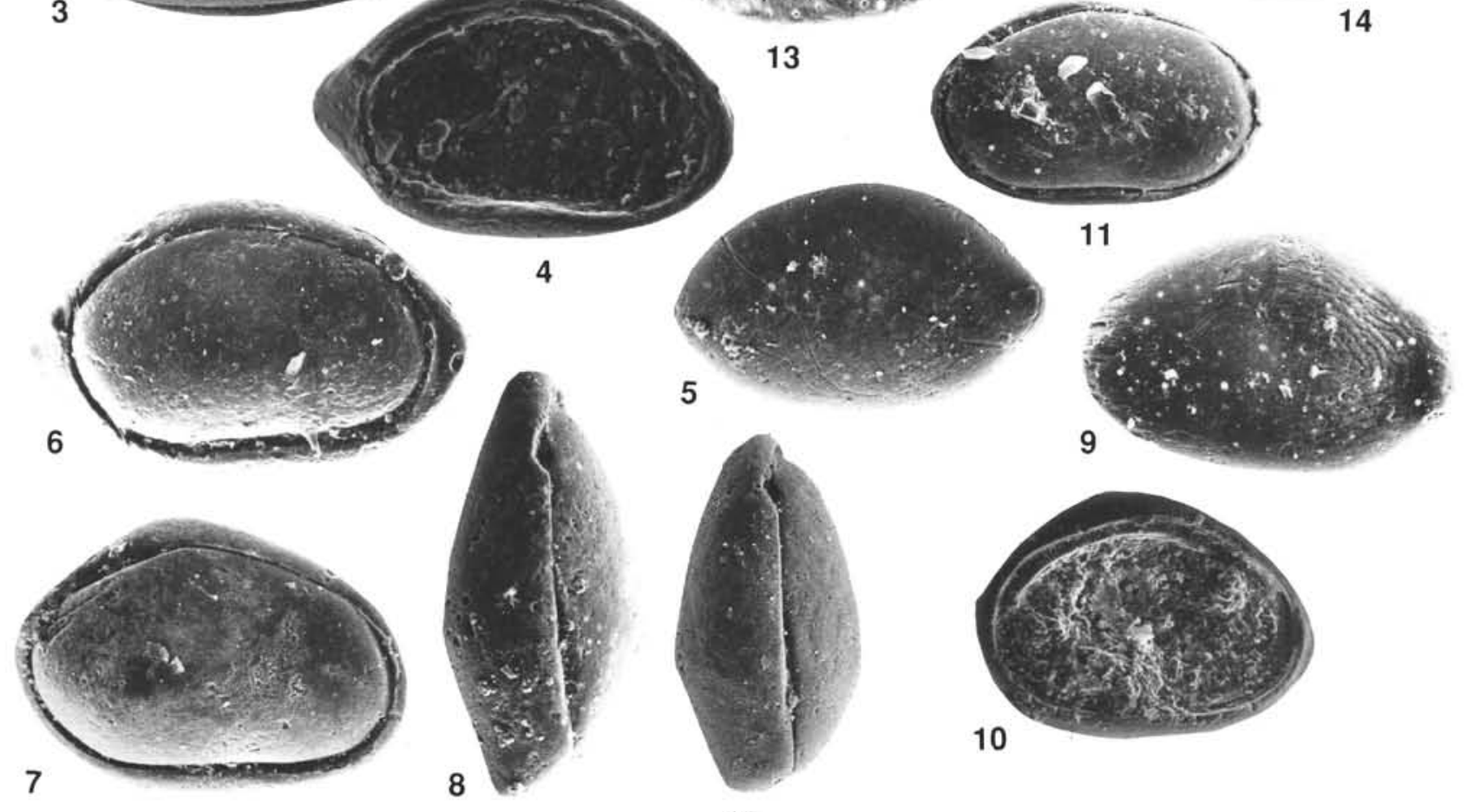

15

Plate 4. Magnification is $80 \times$ unless otherwise stated. 1. Ogmoconcha sp., left view, $95 \times$, Section 122-760B-8R-3. 2. Ogmoconcha cf. blakei (Sohn, 1968), left view, Section 122-760B-9R-2. 3. Ogmoconcha bristolensis (Anderson, 1964), right view, Section 122-764B-31R-5. 4-6. Ogmoconcha cf. reticulata Kristan-Tollmann (1980); (4) left view, 95×; (5) left view; (6) right view, 95×, Section 122-760B-8R-3. 7. Ogmoconcha sp., right view, Section 122-760B-8R-3. 8-10, 15. Ogmoconcha martini (Anderson, 1964); (8) dorsal view, Section 122-760-8R-3; (9) left view, Section 122-764B-31R-5; (10) left valve, internal view, 90×, Section 122-760B-8R-3; (15) dorsal view, Section 122-760B-8R-3. 11. Ogmoconcha sp., right view, Section 122-760B-8R-3. 12. Rhombocythere penarthensis Anderson (1964), left view, 100×, Section 122-760B-8R-3. 13, 14. Rhombocythere penarthensis? Anderson (1961), (13) left view, Section 122-760B-9R-2; (14) right view, Section 122-760B-9R-2. 

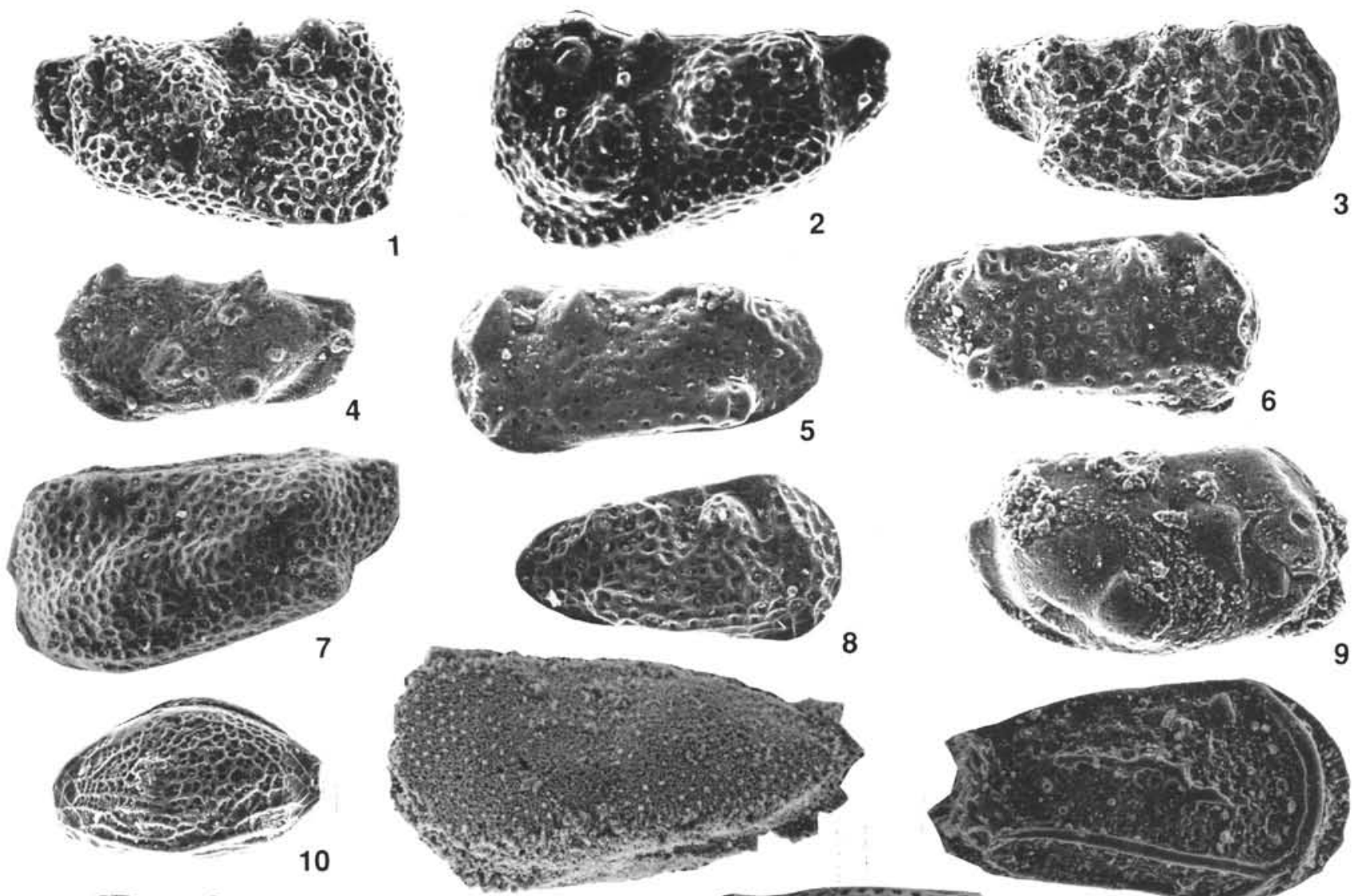

10

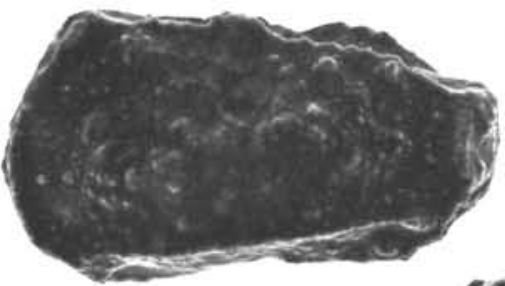

11
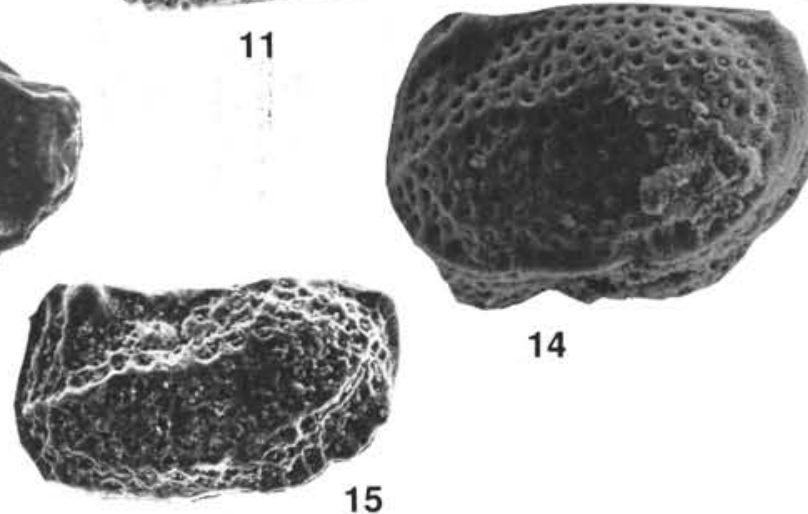

14

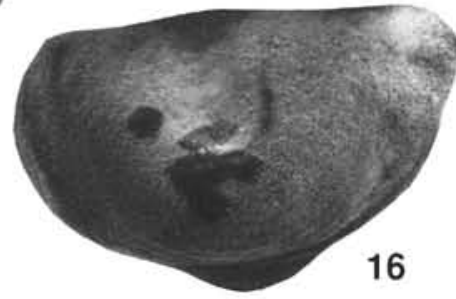

Plate 5. Magnification is $80 \times$ unless otherwise stated. 1, 2. Mostlerella n. sp. 11, right view, 100× (2) left view, 100×, Section 122-760B-8R-3. 3. Mostlerella sp., right view, Section 122-764B-31R-5. 4. Judahella galli Kozur and Bolz (1971), left valve, 100×, Section 122-764B-31R-5. 5, 6. Judahella cf. andrusovi Kozur and Bolz (1971); (5) right view, 100×, Section 122-761C-24R-3; (6) left view, 90×, Section 122-761C-24R-3. 7. Judahella n. sp. 1, left view, Section 122-759B-13R-1. 8. Judahella? sp. 1, right view, 100×, Section 122-764B-11R-2. 9. Indet. gen.? sp., right view, Section 122-759B-11R-2. 10. Kinkelinella? sp. 1, left view, 100×, Section 122-764B-31R-5. 11. Kerocythere cf. norica Bolz and Kozur (1971), left view, Section 122-761C-23R-1. 12. Noricythere n. sp. aff. mosstleri Bolz and Kozur (1971), right view, Section 122-761C-31R-3. 13. Kerocythere sp. A, left view, 70×, Section 122-761C-23R-1. 14. Simeonella? sp. 1, left view, Section 122-759B-13R-1. 15. Mockella cf. muelleri Bunza and Kozur (1971), left view, 100×, Section 122-761C-24R-3. 16. Indet. gen., carapace in left view, 90×, Section 122-764B-8R-1. 